\title{
In-situ high-temperature domain structures of die-upset Nd-Fe-B magnets
}

\author{
YikunFang $^{\mathrm{a}, \mathrm{b}}$, XiaoluYin $^{\mathrm{a}}$, Ralph Skomski ${ }^{\mathrm{a}}$, HongshengCheng ${ }^{\mathrm{b}}$, KuikuiSong $^{\mathrm{b}}$, MinggangZhu $^{\mathrm{b}}$, \\ ZhaohuiGuo $^{\text {b }}$, Wei Li ${ }^{\text {b }}$, Sy-Hwang Liou ${ }^{\mathrm{a}}$ \\ a Department of Physics and Astronomy and Nebraska Center for Materials and Nanoscience, \\ University of Nebraska- Lincoln, Lincoln, NE 68588, USA \\ ${ }^{\mathrm{b}}$ Division of Functional Materials research, Central Iron and Steel Research Institute, Beijing \\ 100081, China
}

Magnetic domains of a die-upset Nd-Fe-B magnet with an energy product of about 50 MGOe are investigated by using in-situ magnetic force microscopy (MFM), varying temperature from room temperature to $240{ }^{\circ} \mathrm{C}$. For both as-prepared and magnetized Nd-Fe-B samples, the domainpattern evolution during thermal demagnetization is monitored by MFM. The temperature dependence of the evolution of reverse domains is explained by micromagnetic models. At all temperatures, the magnetization reversal in the technological relevant magnetized limit proceeds by nucleation.

Keywords: Die-upset Nd-Fe-B magnets; Magnetic force microscopy (MFM); Magnetic domains

The large demand for hybrid electric vehicles and wind turbines for electricity generation has led to renewed interest in the development of Nd-Fe-B magnets, particularly concerning their performance at elevated temperatures $\left(T>150{ }^{\circ} \mathrm{C}\right)$ [1].The understanding of the temperature dependence of domain structures in $\mathrm{Nd}_{2} \mathrm{Fe}_{14} \mathrm{~B}$ is of great importance, because $\mathrm{Nd}_{2} \mathrm{Fe}_{14} \mathrm{~B}$ has a relatively low Curie temperature $\left(T_{\mathrm{c}}=312^{\circ} \mathrm{C}\right)$. The magnetocystalline anisotropy decreases rapidly as the temperature approaches $T_{\mathrm{c}}$ [2], accompanied by a reduction in coercivity and by changes in domain structure and reversal mechanism. It is great of interest to investigate the high temperature domain structures in these materials from both fundamental research and practical application points of views.

To fully exploit the potential of $\mathrm{Nd}_{2} \mathrm{Fe}_{14} \mathrm{~B}$ [3-6], it is necessary to have the magnetic grain $c$ axis aligned. One method of achieving this goal is die-upsetting, where the $c$-axis of the grains is stress-induced and parallel to the press direction [7-11] and where the texture formation is caused by preferential grain growth via dissolution and precipitation[12-14].

Previous studies of the die-upset Nd-Fe-B have shown that the magnetic domains at room 
temperature, referred to as interaction domains, are much larger than the grain size[15-17], because the grains are coupled due to intergranular exchange. The grains included in the interactiondomain are almost in single-domain because their size $(50-400 \mathrm{~nm})$ is comparable to the single domain grain size for $\mathrm{Nd}_{2} \mathrm{Fe}_{14} \mathrm{~B}$ [18].

Many high-performance permanent magnets, for example in motors, operate above room temperature. It is therefore desirable to directly observe domain structures at elevated temperatures, rather than making indirect conclusions, for example from hysteresis loops. Magnetic force microscopy (MFM), a surface technique, has been widely used to reveal the magnetic domains of bulk permanent materials [17,19-24]. Surprisingly, it has not been possible so far to record in-situ MFM images of die-upset Nd-Fe-B magnet above room temperature. In this paper, we directly monitor the magnetic domains of a bulk die-upset Nd-Fe-B magnet at elevated temperature.

The die-upset Nd-Fe-B magnets were prepared by using hot compaction and subsequent hot deformation. Melt-spun powder of $\mathrm{Nd}_{13.62} \mathrm{Fe}_{75.70} \mathrm{Co}_{4.45} \mathrm{~B}_{5.76} \mathrm{Ga} 0.47$ was hot-pressed in vacuum at $550{ }^{\circ} \mathrm{C}$ and $113 \mathrm{MPa}$ to obtain pieces of fully dense isotropic precursors. The precursors were hotdeformed in argon at temperatures from $600{ }^{\circ} \mathrm{C}$ to $900{ }^{\circ} \mathrm{C}$, to achieve a height reduction of $70 \%$. Cylindrical samples approximately $6 \mathrm{~mm}$ in diameter and $4 \mathrm{~mm}$ in height were cut from the center of die-upset magnets, and then their magnetic properties were measured parallel to the loading direction at room temperature, using a hysteresograph. The microstructure of the magnets was studied by field-emission scanning-electron microscopy (SEM), and the crystal structure of the magnets was investigated by x-ray diffraction (XRD) with $\mathrm{Cu} \mathrm{K}_{\alpha}$ radiation. The temperature dependence of saturation magnetization of the samples was measured by vibrating sample magnetometry (VSM) in a magnetic field of $30 \mathrm{kOe}$. The samples for the MFM experiments are cubes of approximately $4 \times 4 \times 4 \mathrm{~mm}^{3}$ taken from central region close to that used for the magnetic measurements. One surface of each of the cubes, with the surface normal in the same direction as the loading direction, was polished with standard metallographic polished techniques using a water-free lubricant.

Figure 1(a) shows the demagnetizing curve in the second quadrant of the hysteresis loop. The remanence $B_{r}$, intrinsic coercivity $H_{c j}$, and maximum energy product $(B H)$ max are $14.2 \mathrm{kGs}$, $12.9 \mathrm{kOe}$, and $50 \mathrm{MGOe}$, respectively. This energy product equals the previous energy-product record for die-upset Nd-Fe-B, achieved by T. Satioet al.[10] and W. Q. Liu et al.[11]. The inset in Fig. 1(a) is the XRD pattern of the die-upset Nd-Fe-B along the loading direction. Reflections 
such as (006), (105), (004) and (008) become dominate and the relative intensity ratio of $(006) /(105)$ is about 1.86 , indicating a strong $c$-axis crystallographic alignment in the magnets. The microstructure of the sample perpendicular to the loading direction [Fig. 1 (b)] consists of elongated nanocrystalline grains with their length perpendicular to the loading direction. Their width and length are about $60 \mathrm{~nm}$ and $400 \mathrm{~nm}$, respectively. The strong $c$-axis alignment and the fairly regular microstructure are one of the reasons for the excellent magnetic properties of the magnet.

To image the magnetic domains of the samples, we have used a Digital Instruments EnviroScope MFM operating in the tapping/lift mode and equipped with a downwardsmagnetized highly coercive Fe-Pt tip [23]. The sample stage of the EnviroScope MFM consists of a thermoelectric unit capable of varying the stage temperature $T$ from room temperature $\left(\sim 23{ }^{\circ} \mathrm{C}\right)$ to about $300{ }^{\circ} \mathrm{C}$. The MFM chamber was pumped down to about $6.7 \times 10^{-3} \mathrm{~Pa}$ to avoid the oxidation of the sample.

In our experiments, we found that the magnetization direction and magnitude of the MFM tips is not significantly changed below $200{ }^{\circ} \mathrm{C}$. Therefore, we carried on our in-situ MFM experiments only below $200{ }^{\circ} \mathrm{C}$. For higher temperatures, between $200{ }^{\circ} \mathrm{C}$ and $300{ }^{\circ} \mathrm{C}$, the sample was first heated to the corresponding temperature and then cooled down to $180{ }^{\circ} \mathrm{C}$ and the MFM images were captured at the same scan area. All MFM images were obtained by the same tip with a liftheight of $50 \mathrm{~nm}$. The average domain width was measured using the stereological method proposed by Bodenberger and Hubert $[25,26]$.

We have captured in-situ MFM images of the as-prepared (thermal demagnetized) die-upset $\mathrm{Nd}-\mathrm{Fe}-\mathrm{B}$ permanent magnet at room temperatureand at $180^{\circ} \mathrm{C}$ in zero applied magnetic field (see Fig.2). The as-prepared sample is almost in a virgin state (thermal demagnetized). The two images are taken by the same tip and for almost the same area. The root-mean-square values of the phase shift, $\phi_{\mathrm{rms}}$, of the Figs.2(a) and 2 (b) are about $12.0^{\circ}$ and $6.25^{\circ}$, respectively. The domain patterns between room temperature and $180^{\circ} \mathrm{C}$ are exactly same as those of regular maze pattern, but show weaker contrast at higher temperature. The average domain width $(w)$ remains $530 \mathrm{~nm}$, which is much larger than the grain size. Consequently, many grains participate in the formation of an interaction domain in which the grains exhibit the similar orientation of magnetization. The weaker contrast is probably due to the decreased magnetization of magnet and MFM tip at high temperature.

The domain pattern reflects the competition between magnetostatistic energy and domain wall 
energy. As assuming the reverse domain is conical with height of $h$ and radius of $b$, the corresponding energy gain $(E)$ on domain formation is as follows:

$$
E=-\frac{1}{3} \pi b^{2} h \mu_{0} M_{s}^{2}+\pi \gamma b\left(\sqrt{b^{2}+h^{2}}\right)
$$

The first term represents magnetostatic energy with $\mathrm{M}_{\mathrm{s}}$ (the saturation magnetization), and the second term represents domain wall energy with $\gamma$ (the domain wall energy density). The relation between $b$ and $h$ is obtained by minimizing the energy with respect to $b, \partial E / \partial b=0$. This yields $b$ $=\sqrt{6} \pi / / \mu_{\mathrm{o}} \mathrm{M}_{\mathrm{s}}{ }^{2}$, which means the corresponding domain size is as large as $2 b=214 \mathrm{~nm}$. The calculated domain size is significantly smaller than the MFM domain size (Fig. 2), which greatly depends on the real structure and sample history. One example is the fully magnetizedstate, where the domain size should approach infinity. This is because domain size is, by definition, an equilibrium property. In non-equilibrium, states with much larger domain size can be stabilized by micro-magnetic energy barriers. The height of the energy barriers is largely determined by the magnetocystalline anisotropy $K_{1}$. Other considerations in explaining the larger actual domain size are the underestimation of the magnetostatic self-interaction in the calculation and the formation of branch domains [26].

In order to investigate the high-temperature domain structures, we studied the sample in the remanent state, which was obtained by magnetizing the as-prepared sample to a field of $70 \mathrm{kOe}$ along the loading direction (easy axis) and then gradual reducing to zero magnetic field. Figures 3 (a-f) show the domain patterns of the magnetized die-upset Nd-Fe-B bulk sample at different temperatures. These in-situ magnetic domain images were captured at the same area. As shown in Fig. 3(a), the magnetization of the sample is nearly fully aligned, so it is virtually free of domains at room temperature. With increasing the temperature, the reverse domains start to show up at 80 ${ }^{\circ} \mathrm{C}$ with size ranging about $1 \mu \mathrm{m}$, and then grow and spread throughout the sample. The facilitated nucleation of reverse domains is probably caused by the rapid weakening of magnetocystalline anisotropy in the magnet, especially at the grain boundaries. For the same reason, the feature size of the reverse domain become slender as the temperature increases. The width of the reverse domains shrinks from $1 \mu \mathrm{m}$ (at $80{ }^{\circ} \mathrm{C}$ ) to $400 \mathrm{~nm}$ (from $160^{\circ} \mathrm{C}$ to $240{ }^{\circ} \mathrm{C}$ ). Note that the direct creation of magnetic domain by thermal activation is extremely unlikely, because $K_{1} V$ is huge, even at elevated temperatures, where $K_{1}$ is relatively small.

The magnetized state is quite different from the as-prepared (thermal demagnetized) state. The large positive magnetic fieldof about $70 \mathrm{kOe}$ removes all the domain structures and keeps the 
sample far away from equilibrium state. This nonequilibrium state is stabilized by the large magnetocrystalline anisotropy, which competes with the demagnetizing energy. The nucleation of reverse domains is therefore determined by the competition between the local nucleation field or effective anisotropy field $2 K_{\mathrm{eff}} / \mu_{\mathrm{o}} M_{\mathrm{s}}$ and the local demagnetizing field $D_{\mathrm{L}} M_{\mathrm{s}}$. In another words, the reverse domain starts to show up when the demagnetizing field overcomes nucleation field,

$$
D_{L} M_{s} \geq \frac{2 K_{e f f}(T)}{\mu_{0} M_{s}}
$$

$D_{L}$ is the pre-factor of local demagnetizing field. In our case, we assume it to be $1 / 3$. $K_{\text {eff, the }}$ effective anisotropy, usually equals from 0.1 to $0.3 K_{1}$ at room temperature. The condition (2) is usually satisfied at high temperatures, since $M_{\mathrm{s}}$ of Nd-Fe-B decreases slowly whereas $K_{1} \sim K_{\text {eff }}$ rapidly decrease with increasing temperature, as shown in Fig. 4. Approximately, $K_{1} \sim M_{\mathrm{s}}{ }^{3}$ [2]. At $80^{\circ} \mathrm{C}$, the demagnetizing field overcomes the nucleation field and the reverse domain appear. During the thermal demagnetization process, two effects compete. On the one hand, the reverse domains grow from the originally rather small nucleus, which directly enhances $D_{\mathrm{L}}$. On the other hand, there may be more than one nucleus, and the reverse domains become themselves magnetostatically unfavorable, promoting a branching of the domains and reducing the domain size.

In conclusion, we have used magnetic force microscopy to in-situ investigate magnetic domain evolution during thermal demagnetization in both as-prepared and magnetized samples of die-upset Nd-Fe-B. In the as-prepared sample, the maze domain pattern remains the same as the temperature increases. By comparison, in the magnetized sample, the reverse domains are removed at room temperaturebut reappear at $80^{\circ} \mathrm{C}$ and get more slender as the temperature increases. This behavior is explained by a nucleation-type thermal demagnetizing process, which reflects the temperature-dependent competition between the local demagnetizing field and anisotropy field.

This work is partly supported by DOD (ARO) W911NF-08-1-01311, W911NF-10-2-0099, NSF MRSEC DMR-0820521, the National Natural Science Foundation of China (51371054, 51331003), and the National Basic Research Program of China (2014CB643701).

[1] T.G. Woodcock, O. Gutfleisch, Acta Mater. 59(2011)1026.

[2] R. Skomski and J. M. D. Coey, Permanent Magnetism, Institute of Physics, Bristol 1999. 
[3] J. J. Croat, J. F. Herbst, R. W. Lee, F. E. Pinkerton, Appl. Phys. Lett. 44 (1984) 148.

[4] M. Sagawa, S. Fujimura, N. Togawa, H. Yamamoto, Y. Matsuura J. Appl. Phys. 55(1984) 2083.

[5] J. F. Herbst, Rev. Mod. Phys. 63(1991) 819.

[6] S. Sugimoto, J. Phys. D: Appl. Phys. 44 (2011)064001.

[7] R. W. Lee, Appl. Phys. Lett. 46 (1985)790.

[8] C. D. Fuerst, E. G. Brewer, J. Appl. Phys. 73 (1993) 5751.

[9] T. Harada, M. Fujita, T. Kuji, J. Alloy. Compd. 243 (1996)139.

[10] T. Saito, M. Fujita, T. Kuji, K. Fukuoka, Y. Syono, J. Appl. Phys. 83 (1998)6390.

[11] W. Q. Liu, Z. Z. Cui, X. F. Yi, M. Yue, Y. B. Jiang, D. T. Zhang, J. X. Zhang, X. B. Liu, J. Appl. Phys. 107 (2010) 09A719.

[12] U. Li, C. D. Graham, Jr., J. Appl. Phys. 67 (1990) 4756.

[13] U. Li, C. D. Graham, Jr., IEEE Trans. Magn. 28 (1992) 2130.

[14] S. Guruswamy, Y. R. Wang, V. Panchanathan, J. Appl. Phys. 83(1998) 6993.

[15] K. Khlopkov, O. Gutfleisch, R. Sch.afer, D. Hinz, K.-H. Müller, L. Schultz, J. Magn. Magn. Mater. 272 (2004) e1937.

[16] K. Khlopkov, O. Gutfleisch, D. Hinz, K.-H. Müller, L. Schultz, J. Appl. Phys. 102(2007) 023912.

[17] Y. K. Fang, X. L. Yin, R. Zhao, S. Valloppilly, W. Li, M. G. Zhu, S. H. Liou, J. Appl. Phys. 111 (2012) 07A734.

[18] K. H. J. Buschow, Mater. Sci. Rep. 1 (1986) 1.

[19] W. Szmaja, J. Grobelny, M. Cichomski, Appl. Phys. Lett.85 (2004) 2878.

[20]M. G. Zhu, Y. K. Fang, Y. Q. Guo, W. Li, B. S. Han, J. Magn. Magn. Mater. 282 (2004) 180.

[21]O. Gutfleisch, K.-H.Müller, K. Khlopkov, M. Wolf, A. Yan, R. Schäfer, T. Gemming, L. Schultz, ActaMater. 54 (2006) 997.

[22]Y. K. Fang, H. W. Chang, Z. H. Guo, T. Liu, X. M. Li, W. Li, W. C. Chang, B. S. Han, J. Alloys Compds. 462 (2008) 376.

[23] Y. K. Fang, L. Yang, W. Li, L. Zhen, B. S. Han, J. Magn. Magn. Mater. 322 (2010) 3270.

[24]W. Szmaja, J. Grobelny, M. Cichomski, S. Hirosawa, Y. Shigemoto, Acta Mater. 59 (2011) 531.

[25] R. Bodenbergerand A. Hubert, phys.stat.sol. (a) 44 (1977) K7.

[26] A. Hubert, R. Schäfer, Magnetic Domains-The Analysis of Magnetic Microstructures (Springer, Berlin, 1998). 
[27] Yu.G. Pastushenkov, A. Forkl, H. Kronmüller, J. Magn. Magn. Mater. 174 (1997) 278.

\section{Figure captions:}

Figure 1.Die-upset Nd-Fe-B sample: (a) demagnetization curve at room temperature and (b) SEM image along the loading direction (marked by arrows). The inset in (a) is the XRD pattern of the sample.

Figure 2. Magnetic domains in a $10 \times 10 \mu \mathrm{m}^{2}$ area of the as-prepared sample: (a) at room temperature and (b) at $180{ }^{\circ} \mathrm{C}$. The root-mean-square values of the phase shift, $\phi_{\mathrm{rms}}$, of the images (a) and (b) are about $12.0^{\circ}$ and $6.25^{\circ}$, respectively, indicating the saturation magnetization is substantially smaller at $180{ }^{\circ} \mathrm{C}$ than at room temperature. The circles show the same area of the images. The loading direction (easy axis) is perpendicular to the image plane.

Figure 3. MFM images of one $20 \times 20 \mu \mathrm{m}^{2}$ of the sample at different temperatures: (a) $23{ }^{\circ} \mathrm{C}$, (b) $80{ }^{\circ} \mathrm{C}$, (c) $120{ }^{\circ} \mathrm{C}$, (d) $160{ }^{\circ} \mathrm{C}$, (e) $180{ }^{\circ} \mathrm{C}$, and (f) heated up to $240{ }^{\circ} \mathrm{C}$ and then cooled to $180{ }^{\circ} \mathrm{C}$. The sample was first magnetized in $70 \mathrm{kOe}$, the loading direction being perpendicular to the image plane, and then imaged at remanence $(H=0)$.

Figure 4.Temperature dependence of the normalized saturation magnetization $M_{\mathrm{s}}(\mathrm{T})$ and of $\left[M_{\mathrm{s}}(\mathrm{T})\right]^{2}$ of the die-upset Nd-Fe-B sample, measured in a magnetic field of $30 \mathrm{kOe}$. The temperature dependence of the normalized magnetic anisotropy constant $K_{1}(\mathrm{~T})$ of a $\mathrm{Nd}_{2} \mathrm{Fe}_{14} \mathrm{~B}$ single crystal [27] is also plotted. The temperature dependence of $\left[M_{\mathrm{s}}(\mathrm{T})\right]^{2}$ is much weaker than that of $K_{1}(\mathrm{~T})$. 

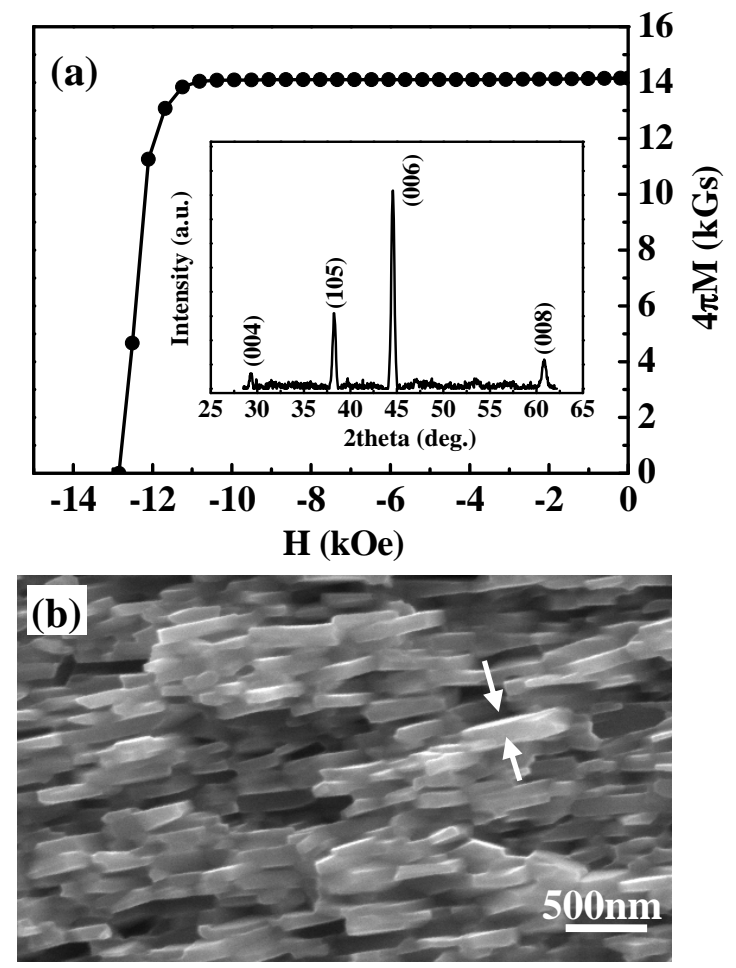

Figure 1.Die-upset Nd-Fe-B sample: (a) demagnetization curve at room temperature and (b) SEM image along the loading direction (marked by arrows). The inset in (a) is the XRD pattern of the sample. 


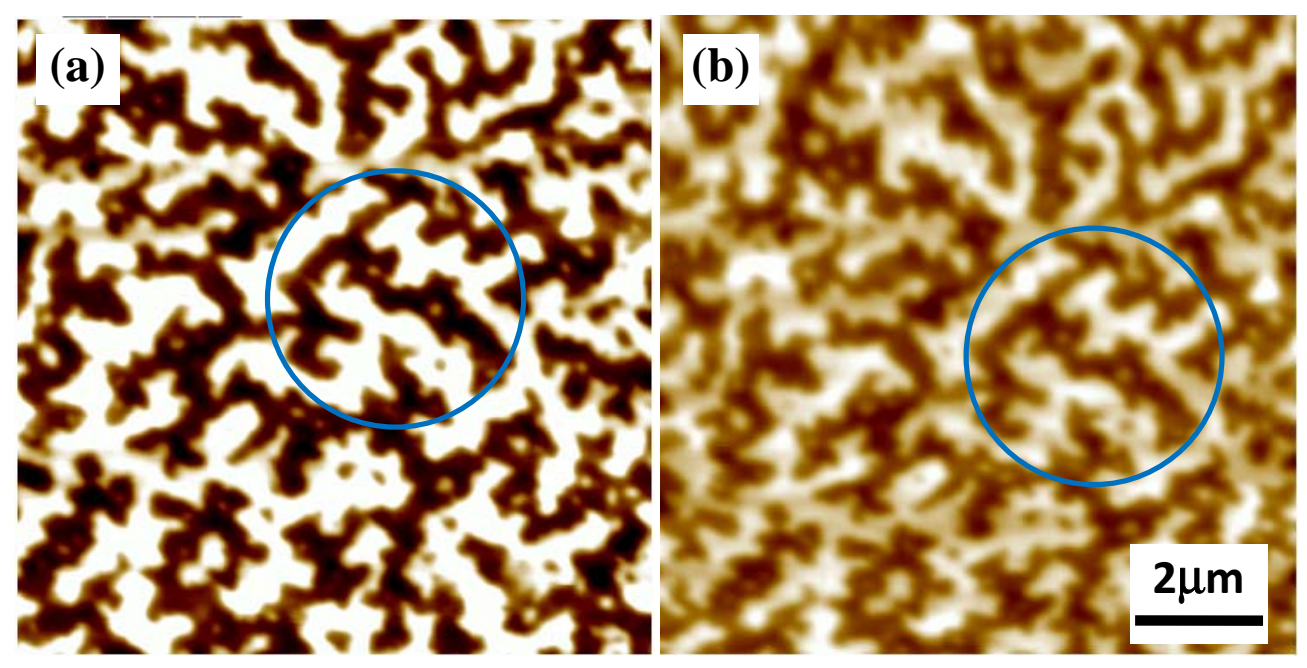

Figure 2. Magnetic domains in $\mathrm{a} 10 \times 10 \mu \mathrm{m}^{2}$ area of the as-prepared sample: (a) at room temperature and (b) at $180{ }^{\circ} \mathrm{C}$. The root-mean-square values of the phase shift $\phi_{\mathrm{rms}}$ of the images (a) and (b) are about $12.0^{\circ}$ and $6.25^{\circ}$, respectively, indicating that the saturation magnetization is substantially smaller at $180{ }^{\circ} \mathrm{C}$ than at room temperature. The circles show the same area of the images. The loading direction (easy axis) is perpendicular to the image plane. 

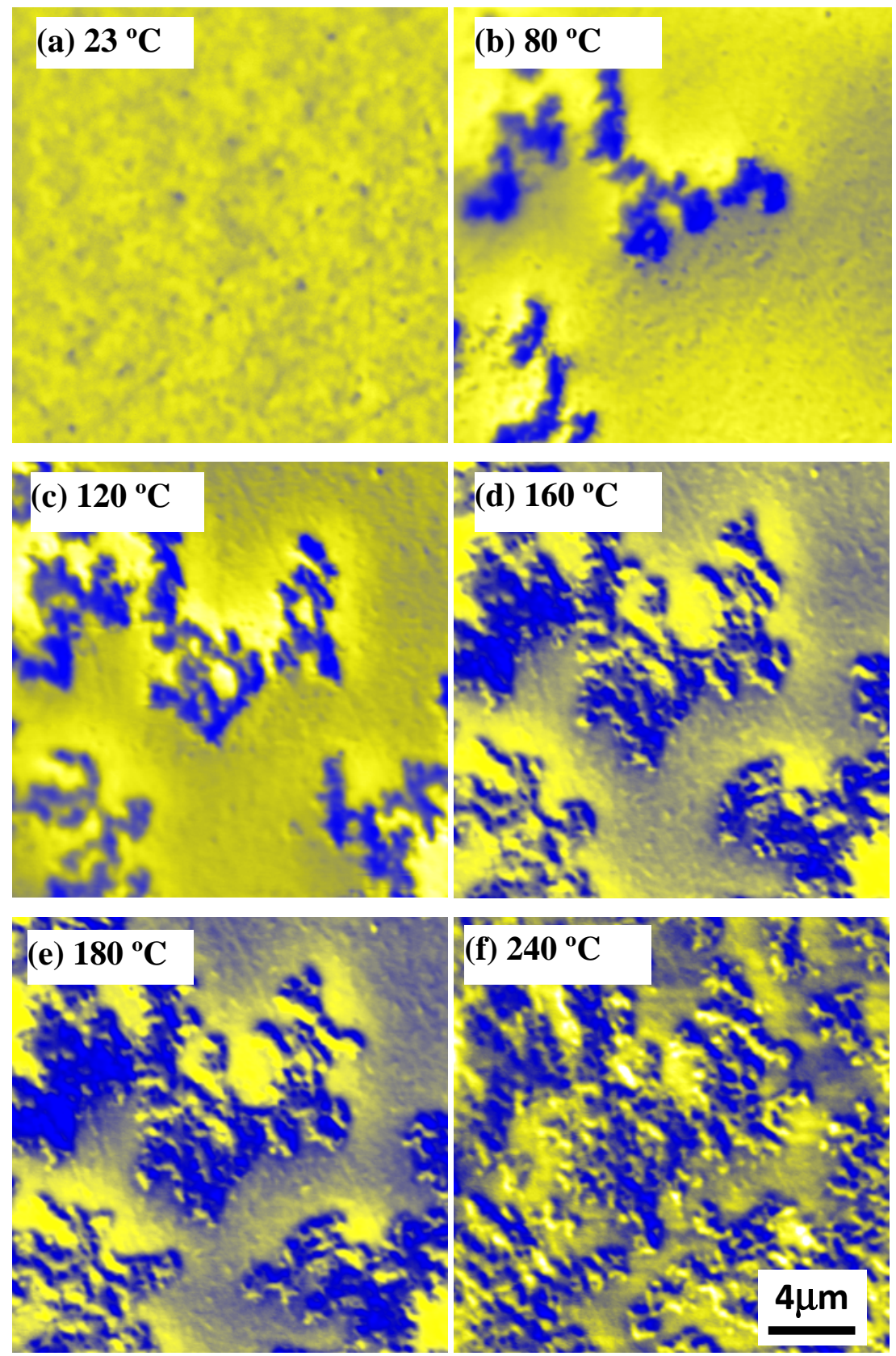

Figure 3. MFM images of one $20 \times 20 \mu \mathrm{m}^{2}$ area of the sample at different temperatures: (a) $23{ }^{\circ} \mathrm{C}$, (b) $80^{\circ} \mathrm{C}$, (c) $120^{\circ} \mathrm{C}$, (d) $160{ }^{\circ} \mathrm{C}$, (e) $180{ }^{\circ} \mathrm{C}$, and (f) heated up to $240{ }^{\circ} \mathrm{C}$ and then cooled to $180{ }^{\circ} \mathrm{C}$. The sample was first magnetized in $70 \mathrm{kOe}$, the loading direction being perpendicular to the image plane, and then imaged at remanence $(H=$ $0)$. 


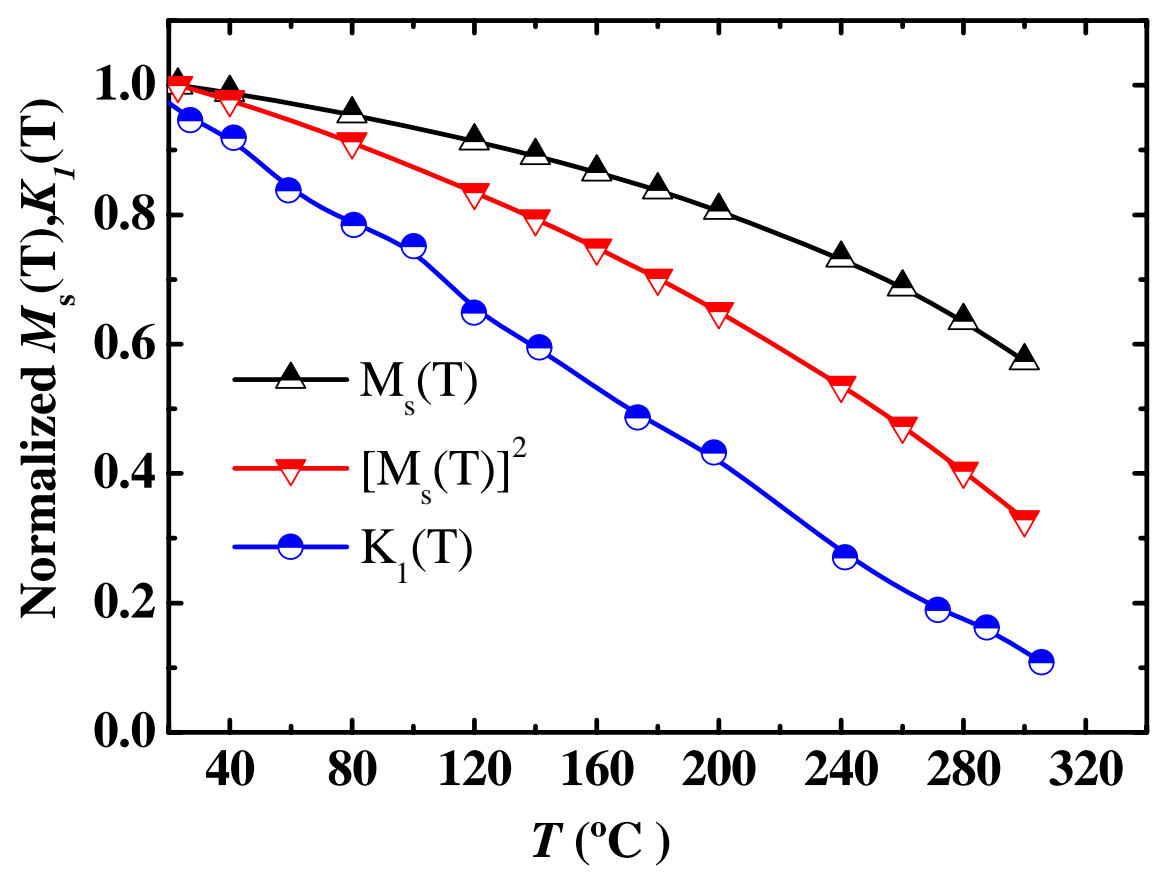

Figure 4. Temperature dependence of the normalized saturation magnetization $M_{\mathrm{s}}(T)$ and of $\left[M_{\mathrm{s}}(T)\right]^{2}$ of the die-upset Nd-Fe-B sample, measured in a magnetic field of 30 kOe. The temperature dependence of the normalized magnetic anisotropy constant $K_{1}(T)$ of a $\mathrm{Nd}_{2} \mathrm{Fe}_{14} \mathrm{~B}$ single crystal [27] is also plotted. The temperature dependence of $\left[M_{\mathrm{s}}(T)\right]^{2}$ is much weaker than that of $K_{1}(T)$. 
Magnetic domains of a die-upset Nd-Fe-B magnet with an energy product of about 50 MGOe are investigated by using in-situ magnetic force microscopy (MFM), varying temperature from room temperature to $240{ }^{\circ} \mathrm{C}$. For both as-prepared and magnetized $\mathrm{Nd}-\mathrm{Fe}-\mathrm{B}$ samples, the domain-pattern evolution during thermal demagnetization is monitored by MFM (Fig. 1). The temperature dependence of the evolution of reverse domains is explained by micromagnetic models. At all temperatures, the magnetization reversal in the technological relevant magnetized limit proceeds by nucleation.
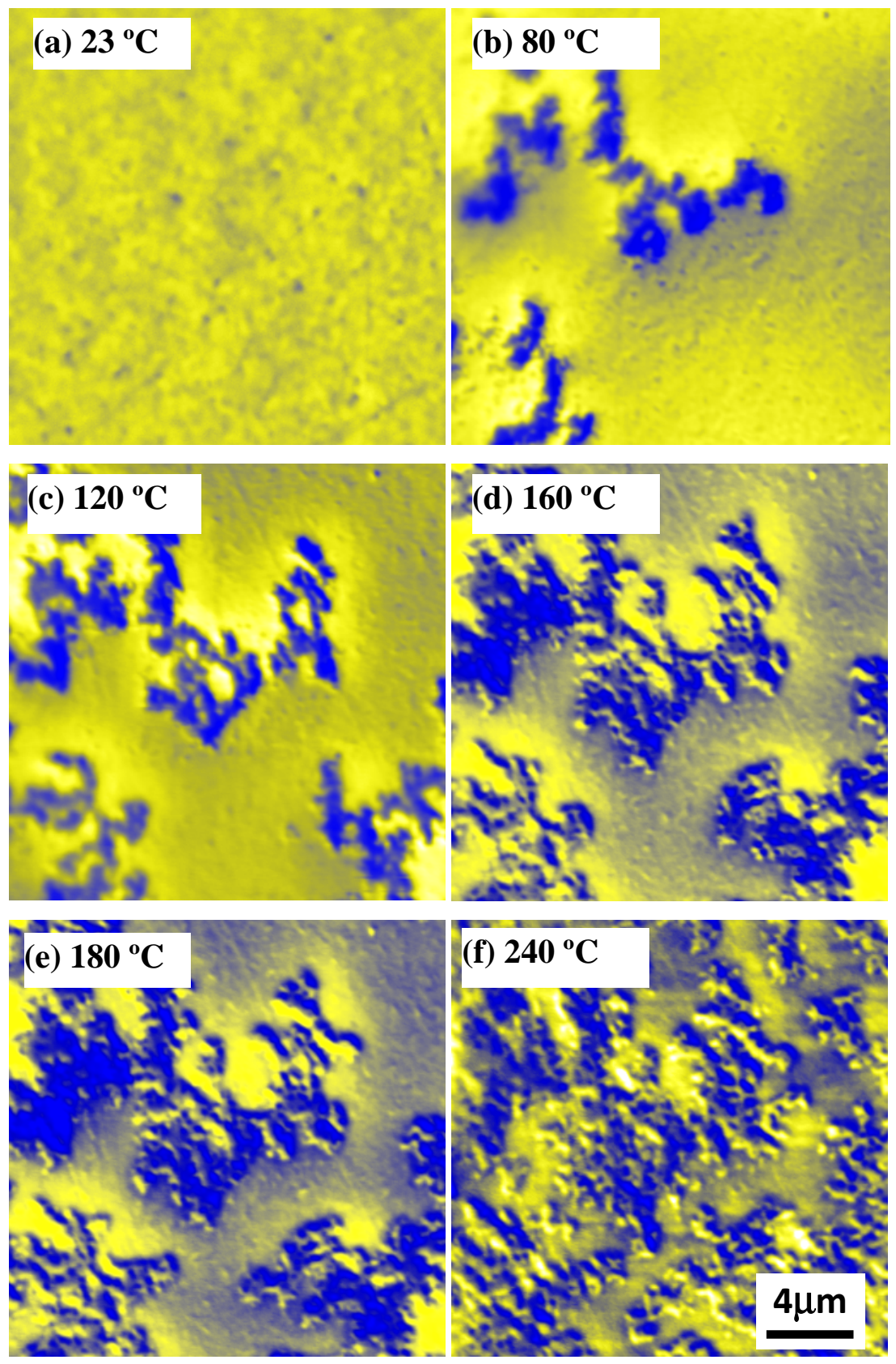

Figure 1. MFM images of one $20 \times 20 \mu \mathrm{m}^{2}$ of the sample at different temperatures: (a) $23^{\circ} \mathrm{C}$, (b) 80 ${ }^{\circ} \mathrm{C}$, (c) $120^{\circ} \mathrm{C}$, (d) $160{ }^{\circ} \mathrm{C}$, (e) $180^{\circ} \mathrm{C}$, and (f) heated up to $240{ }^{\circ} \mathrm{C}$ and then cooled to $180^{\circ} \mathrm{C}$. The sample was first magnetized in $70 \mathrm{kOe}$, the loading direction being perpendicular to the image plane, and then imaged at remanence $(H=0)$. 